Check for updates

Cite this: RSC Adv., 2017, 7, 45061

Received 7th July 2017

Accepted 12th September 2017

DOI: 10.1039/c7ra07511a

rsc.li/rsc-advances

\title{
Practical stability of $\mathrm{Au}_{25}(\mathrm{SR})_{18}{ }^{-1 / 0 /+1_{\dagger}}$
}

\author{
C. B. Collins, (D) a M. A. Tofanelli, ${ }^{\text {b M. F. } \text { Crook, }^{c} \text { B. D. Phillips }}{ }^{\text {a }}$ and C. J. Ackerson*a
}

Superatom electron shell and/or geometric shell filling underlies the thermodynamic stability of coinage and alkali metal clusters in both theoretical and experimental results. Factors beyond simple shell filling contribute substantially to the lifetime of ligated clusters in solution. Such factors include the nature of the solvent, the atmosphere and the steric size of the ligand shell. Here we systematically lay out a 'practical' stability model for ligated metal clusters, which includes both shell-closing aspects and colloidal stability aspects. Cluster decomposition may follow either fusion or fission pathways. Solvent polarity can be determinative of the decomposition pathway.

\section{Introduction}

Gas-phase and solution-phase (ligated) metal clusters comprised of alkali or coinage metals form in discrete, thermodynamically stable sizes for which a possible explanation of is found in superatom complex theory. ${ }^{1}$ This describes molecule-like, discrete electronic states for the delocalized core metal electrons due to quantum confinement of gold nanoclusters with approximately 150 atoms or fewer. According to the superatom model, clusters with closed electronic shells are more stable than those with open electronic shells. Experimentally, we previously showed that superatomic electron configuration predicts the thermal stability of the clusters in differential scanning calorimetry experiments. ${ }^{2}$ In this work, we observed that $\mathrm{Au}_{25}(\mathrm{PET})_{18}{ }^{-1}\left(1 \mathrm{~S}^{2} 1 \mathrm{P}^{6}\right)$ was more thermally stable than $\mathrm{Au}_{25}(\mathrm{PET})_{18}{ }^{0}\left(1 \mathrm{~S}^{2} 1 \mathrm{P}^{5}\right)$ or $\mathrm{Au}_{25}(\mathrm{PET})_{18}{ }^{+1}\left(1 \mathrm{~S}^{2} 1 \mathrm{P}^{4}\right)$.

While superatom electron theory offers explanations for the discrete sizes $^{3-5}$ and thermal stability of the clusters, little investigation has been done into the practical, solution stability of the monolayer protected clusters. ${ }^{6-8}$ This is an area that has been well studied for larger colloidal particles, ${ }^{9-12}$ but it is not clear whether the same principles apply to the smaller particles, and how colloidal descriptions of stability and superatomic descriptions of stability interact.

When a metal cluster or particle 'decomposes', that decomposition can be by fission/etching (breaking into smaller component pieces) or fusion (agglomerative growth of particles). Fission is an established pathway of decay for thiolate protected silver clusters. ${ }^{7}$ Etching represents a type of fission

\footnotetext{
a Department of Chemistry, Colorado State University, Fort Collins, Colorado 80523, USA.E-mail: Ackerson@colostate.edu

${ }^{b}$ Department of Chemistry, University of Pittsburgh, Pittsburgh, Pennsylvania 15260, USA

${ }^{c}$ Department of Chemistry, Binghamton University, Binghamton, New York 13902, USA $\uparrow$ Electronic supplementary information (ESI) available: Decay spectra and trend fits. See DOI: $10.1039 / \mathrm{c} 7 \mathrm{ra07511a}$
}

that is widely used in synthesis of metal nanostructures and is highly dependent on oxygen in the case of thiol protected gold nanoparticles. ${ }^{13-16}$ Instability through agglomerative growth pathways for nanoparticles is well established for water-soluble colloids ${ }^{17}$ and also for organo-soluble colloids. ${ }^{18,19}$ Theoretically, colloidal stability is attributed to a combination of van der Waals attraction, electrostatic double layer repulsion, and steric terms, described classically in DLVO and extended DLVO theory. ${ }^{17,20}$ For water soluble nanoclusters, manipulation of ligand shell charge has been shown to improve solution stability.,21

In this work, we endeavour to establish the "practical stability" of thiolate protected metal clusters, using $\mathrm{Au}_{25}(\mathrm{SR})_{18}$ as a well-studied model system in which different oxidation states and ligand shells are easily accessible. ${ }^{21-28}$ Such practical stability is important to establish as these clusters find their way into catalytic and imaging applications. ${ }^{29,30}$ We find that practical stability appears to depend both on superatom stability concepts and on colloidal stability concepts.

\section{Experimental}

Clusters were synthesized and purified as previously reported. ${ }^{2}$ For air free decomposition studies, the dry cluster was suspended in solvent that was rigorously purged with argon and then transferred to a sealed Schlenk cuvette using Schlenk technique to avoid introduction of air. The cuvettes were sealed to avoid contamination by atmosphere and solvent evaporation. The in air samples were also put into the same Schlenk cuvette but balloons were used to ensure there was a constant atmosphere of air in the head space. UV-vis spectra were taken on an ocean optics usb4000 spectrometer using a DT-mini-2-GS deuterium light source at approximately 4 hour intervals throughout the day using the respective solvent in the same type of cuvette as a blank before each measurement. Concentration of the samples were found using the previously reported molar absorptivity at $680 \mathrm{~nm}$ of $8800 \mathrm{~cm}^{-1} \mathrm{M}^{-1} \cdot{ }^{31}$ Wavelengths at 680 and $605 \mathrm{~nm}$ were monitored so that the trough at 605 could be 
consistently set to zero and the feature at $680 \mathrm{~nm}$ could be compared between measurements. Then the starting concentration was normalized to one and the decrease in concentration was monitored as a decrease in the $680 \mathrm{~nm}$ feature. The concentrations were then plotted $v s$. time to determine the reactant order in $\mathrm{Au}_{25}$ according to the integrated rate laws for zeroth, first and second order reactions. The best fit parameters were then used to determine the rate constant $k$ and the half-life according to the integrated rate law for that given order.

\section{Results and discussion}

We observe that under some conditions, $\mathrm{Au}_{25}(\mathrm{SR})_{18}$ cluster lifetime is extraordinarily longer than under other conditions, where the clusters degrade rapidly. Fig. 1, left panel illustrates this, showing $\mathrm{Au}_{25}\left(\mathrm{SC}_{4} \mathrm{H}_{9}\right)_{18}{ }^{0}$ in rigorously degassed THF, kept solvated under an argon atmosphere in the top set of traces. As judged by the $\mathrm{Au}_{25}\left(\mathrm{SC}_{4} \mathrm{H}_{9}\right)_{18}{ }^{0}$ optical absorbance spectrum, the cluster does not substantially decay over the course of 6 days (spectra are offset for clarity). By comparison, the spectrum of the same compound, when solvated in un-stabilized THF (bottom traces), loses the characteristic linear absorption features of $\mathrm{Au}_{25}\left(\mathrm{SC}_{4} \mathrm{H}_{9}\right)_{18}{ }^{0}$, evolving to a rather featureless spectrum typical of a heterogeneous mixture within the course of 3 days. Mechanistic information about decay pathways is extracted by examining the decrease in absorbance of the spectral feature at $680 \mathrm{~nm}$, attributed to the HOMO-LUMO gap of the $\mathrm{Au}_{25}(\mathrm{SR})_{18}$ cluster. Fitting the evolution of this peak to integrated kinetic rate laws (Table 1) produces information on the order of decay, rate constant, and half-life of the cluster in
Table 1 Integrated rate laws used to determine best fit and reactant order. The $R^{2}$ values here are shown for $\mathrm{Au}_{25}(\mathrm{PET})_{18}{ }^{0}$ in acetone data shown the right panel of Fig. 1

\begin{tabular}{lll}
\hline Reactant order & Integrated rate law fit & $R^{2}$ value \\
\hline Zero (red) & {$[A]=[A]_{0}-k t$} & 0.895 \\
First (blue) & {$[A]=[A]_{0} \mathrm{e}^{-k t}$} & 0.972 \\
Second (not shown) & $\frac{1}{[A]}=\frac{1}{[A]_{0}}+k t$ & 0.0156
\end{tabular}

different environments. An example of this fitting is shown in Fig. 1, right panel.

Many prior reports have noted informally that ligand steric size and/or net charge impacts the practical stability of thiolate protected gold clusters, suggesting that aspects of colloidal stability are important in overall practical stability of thiolate protected clusters. $^{32,33}$ The extent of the influence of ligand size on cluster stability, to our knowledge, has not previously been systematically investigated. To systematically establish the influence of ligand steric size on practical stability of $\mathrm{Au}_{25}(\mathrm{SR})_{18}$, we investigated both the thermal and temporal stability of the compound with $\mathrm{R}$ groups ranging in size from $\left(\mathrm{SCH}_{2} \mathrm{CH}_{3}\right.$, ethanethiol) to $\left(\mathrm{S} \mathrm{CH}_{2}\right)_{11} \mathrm{CH}_{3}$, dodecanethiol), as well as $\left(\mathrm{SCH}_{2} \mathrm{CH}_{2} \mathrm{C}_{6} \mathrm{H}_{5}\right.$, phenylethanethiol, PET). To determine temporal stability of $\mathrm{Au}_{25}(\mathrm{SR})_{18}$, optical spectra of the clusters were acquired at approximately 4 hour intervals over the length of the experiment. The temporal stability in air, shown as half-life of the cluster, for $\mathrm{Au}_{25}(\mathrm{SR})_{18}{ }^{0}$ in dichloromethane solvation.where $\mathrm{SR}=$ ethanethiol, hexanethiol, octanethiol, dodecanethiol and phenylethanethiol is shown in Fig. 2, right panel. The thermal stability of a similar set of clusters was determined by differential scanning calorimetry (DSC). We previously used DSC to
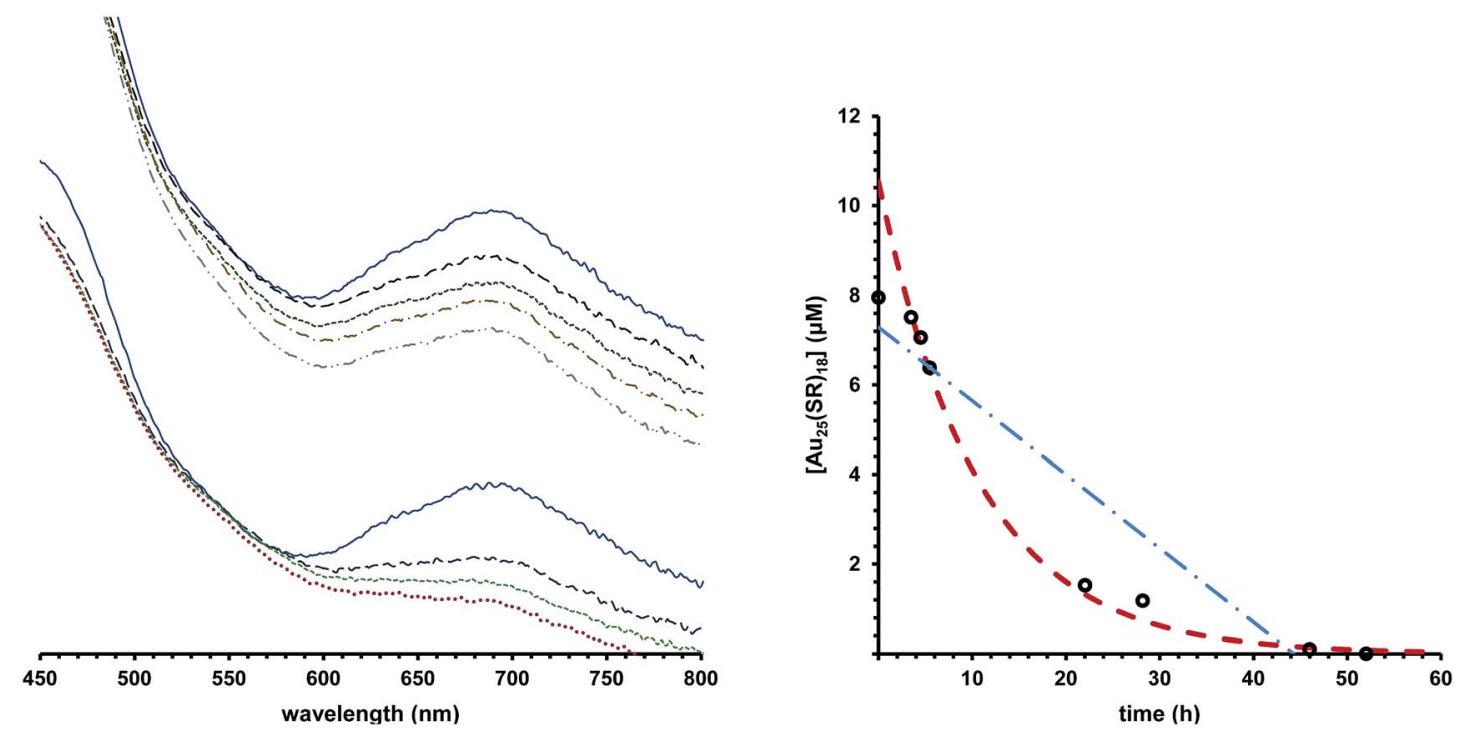

Fig. 1 Left panel shows the optical spectrum of $\mathrm{Au}_{25}\left(\mathrm{SC}_{4} \mathrm{H}_{9}\right)_{18}$ under argon as synthesized (solid blue trace), after approximately 1 day (purpledashed trace), 2 days (green small-dash trace), 3 days (red dot-trace), 5 days (brown dash-dot trace), and 6 days (grey dash-dot-dot trace). The top set of traces show the evolution of the $\mathrm{Au}_{25}\left(\mathrm{SC}_{4} \mathrm{H}_{9}\right)_{18}{ }^{-1}$ spectrum in stabilized THF, while the bottom set of traces shows the same experiment in unstabilized THF. The right panel plots the decay of $\mathrm{Au}_{25}(\mathrm{PET})_{18}{ }^{0}$ in acetone THF measuring concentration with $8800 \mathrm{~cm}^{-1} \mathrm{M}^{-1}$ extinction coefficient at $680 \mathrm{~nm}$, black circles. Fits to this data by zeroth order decay and first order decay are shown as blue and red traces, respectively. 

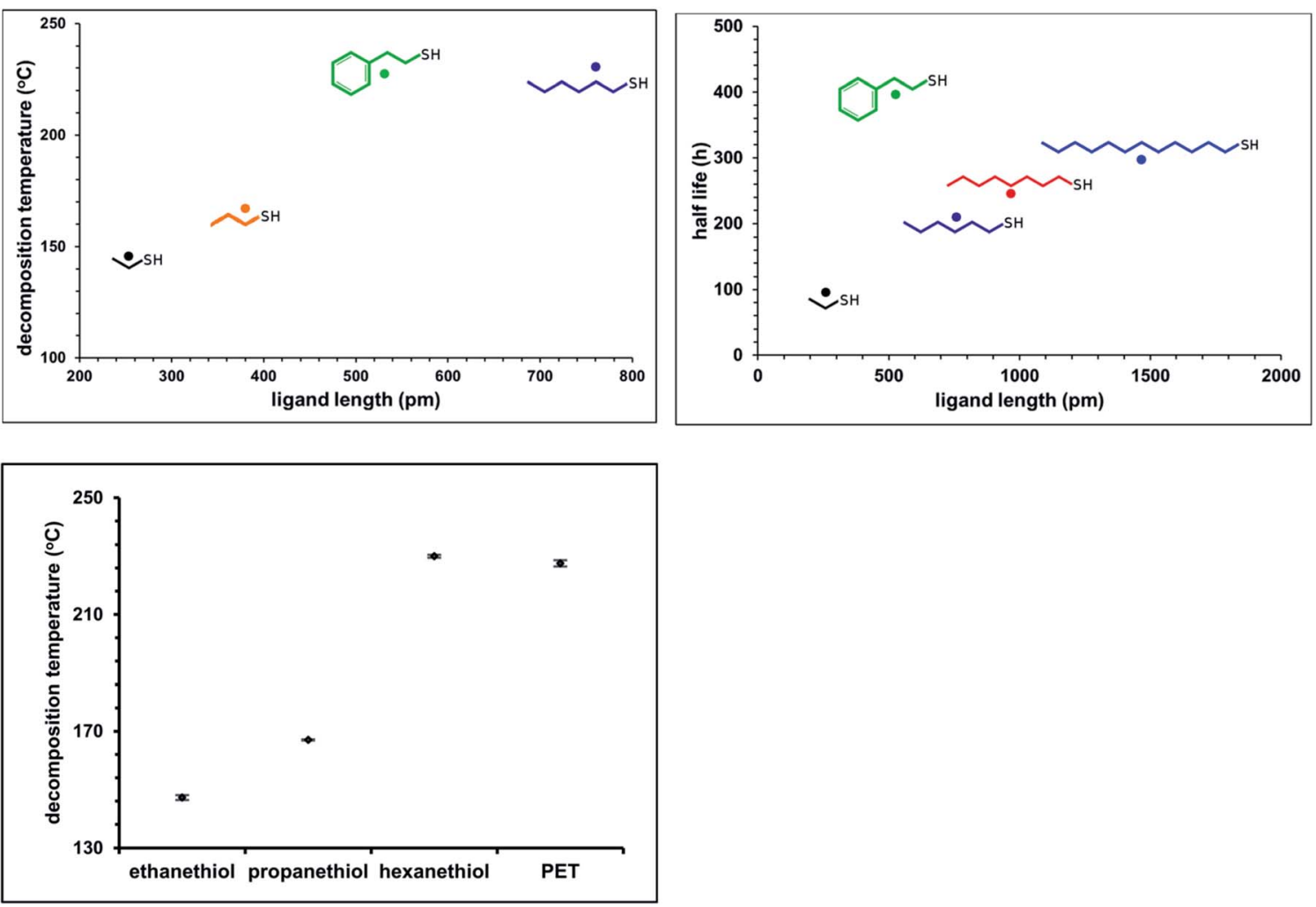

Fig. 2 Left-hand panel shows the thermal stability of the clusters as it related to steric size of the protecting ligand. Right-hand panel shows the temporal stability of $\mathrm{Au}_{25}(\mathrm{SR})_{18}{ }^{\circ}$ as it relates to the steric size of the protecting ligand, air free in DCM.

investigate the stability of $\mathrm{Au}_{25}(\mathrm{PET})_{18}$ clusters as a function of oxidation state. ${ }^{2}$ The raw DSC traces (ESI p S17†) show a large endotherm that we attribute to a decomposition temperature. The decomposition temperatures of clusters protected by ethanethiol, butanethiol, phenylethane thiol and hexanethiol are shown in the right panel of Fig. 2.

Overall, we observe a strong correlation with sterically larger ligands conferring greater thermal and temporal stability. The exception to this trend, phenylethanethiol, is easily rationalized as a more sterically demanding ligand which, by virtue of its branched nature, provides a larger 'cluster cone angle ${ }^{34}$ relative to its length, when compared to straight chain alkanethiols. We expect that branched alkanethiols may also show increased stability relative to absolute chain length.

We also examined the effect of cluster oxidation state on stability. Previously, we suggested that 'closed shell' superatom electron configurations are associated with greater thermal stability. ${ }^{2}$ Here we expand this investigation to examine how the thermal stability of different oxidation states correlates to the steric size of the protecting ligand. Fig. 3 shows the observed decomposition temperatures for $\mathrm{Au}_{25}$ clusters protected by ethanethiol (orange), propanethiol (purple), hexanethiol (blue), and phenylethanethiol (red) in the -1 , and 0 oxidation states. As we observed before, increasing the oxidation state of the clusters, deviating from a full superatomic electron shell generally decreases their thermal stability. Fig. 3 shows that when varied simultaneously, the steric size of the ligand provides a larger contribution than oxidation state to practical thermal stability.

In contrast to the influence of oxidation state on thermal stability of desolvated clusters, we observe a weaker correlation between oxidation state and lifetime in solution for solvated clusters. Instead, we observe that the nature of the solvent appears to dominate the lifetime of the clusters in solution (Fig. 4 and 5).

We also examined the effect storage of $\mathrm{Au}_{25}(\mathrm{SR})_{18}$ in solution under ambient atmosphere compared to in solution under

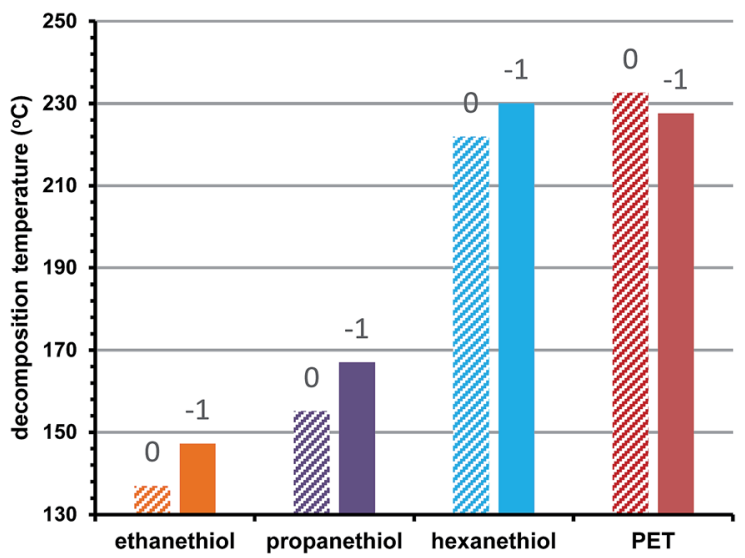

Fig. 3 Oxidation state vs. thermal decomposition temperature. Charge states are shown above each column. 

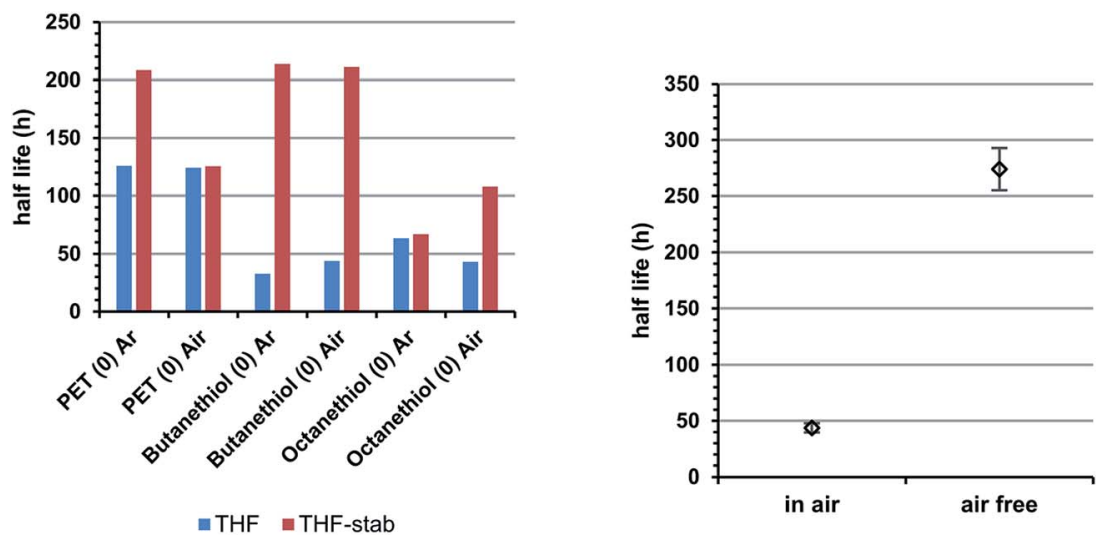

Fig. 4 Left panel shows lifetimes of $\mathrm{Au}_{25}(\mathrm{SR})_{18}{ }^{0}$ in THF and stabilized THF. Generally, the inclusion of BHT increases observed half-life. The right panel shows inclusion vs. exclusion of ambient atmosphere for $\mathrm{Au}_{25}(\mathrm{PET})_{18}{ }^{-1}$ in dichloromethane, showing the lifetime of the cluster is substantially decreased by exposure to atmosphere.

argon. In general, we note that storage under argon preserves cluster integrity for longer periods of time than storage under atmosphere. Fig. 4, right panel shows the lifetime of $\mathrm{Au}_{25}(\mathrm{PET})_{18}$ in DCM under air and under argon. Here, storage under ambient atmosphere notably decreases the half life of the cluster. In the case of THF solvation, however, stabilized THF, which includes a radical inhibitor butylated hydroxytoluene (BHT) allows much longer half lives, independent of the storage atmosphere. That argon atmospheres and BHT specifically both tend to enhance cluster lifetimes is suggestive of dissolved $\mathrm{O}_{2}$ playing a role in the mechanism of cluster decomposition, potentially through an oxygen dependent etching process. ${ }^{13}$

In our preliminary observations, we noted that the nature of the solvent appeared to play a substantial role in the lifetime of solvated $\mathrm{Au}_{25}(\mathrm{SR})_{18}$. We therefore made a systematic investigation of the effect of solvent on the lifetime of the clusters in solution. In testing several solvents used for organo-soluble clusters, we observed a strong correlation between solvent polarity and the lifetime of the cluster in solution, with more polar solvents promoting more rapid decomposition. Fig. 5

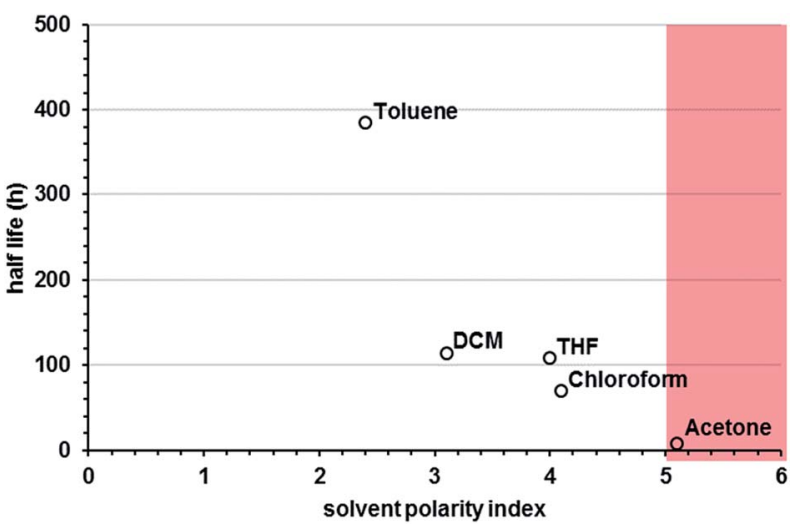

Fig. $5 \mathrm{Au}_{25}(\mathrm{PET})_{18}{ }^{0}$ half lives in toluene, dichloromethane (DCM), tetrahydrofuran, chloroform and acetone in air. The red-overlay represents regions of polarity solvent polarity where fusion mechanisms are (increasingly) observed. shows the half lives of $\mathrm{Au}_{25}(\mathrm{PET})_{18}{ }^{0}$ in a selection of solvents arranged according to their polarity index. ${ }^{35}$ The polarity index is a relative measure of the degree of interaction with a polar test solute, from Burdick \& Jackson solvents. ${ }^{35}$ In general we find that clusters are markedly more stable in toluene than many of the other solvents screened. Some combinations of ligand and solvent, such as butanethiol protected particles in toluene (ESI p S15 $\dagger$ ) resulted in no measurable decay of the particles over 20 days.

This data provides insight into decay pathways. Decay of a suspended particle may arise from one of two either fusion of two particles or fission of a single particle into smaller components. These processes have distinct kinetics, with fission pathways following $0^{\text {th }}$ order kinetics and fusion pathways following $1^{\text {st }}$ order kinetics. An analysis of the disappearance of characteristic peaks of $\mathrm{Au}_{25}$ and spectral evolution into something suggestive of a mixture suggests that the mechanism of decay is environment dependent.

We notice that solvent polarity appears not only determinative of half-life but also influences the mechanism of decay. Higher polarity solvents favor $0^{\text {th }}$ order decay (fission) pathways and lower polarity solvents favor $1^{\text {st }}$ order decay (fusion). Example fits of $0^{\text {th }}$ and $1^{\text {st }}$ order decay are shown the ESI p S12. $\dagger$ This suggests that the higher polarity solvents may be driving aggregation of particles capped by non-polar ligands. We expect to observe the opposite trend for particles capped by polar ligands.

The trends shown are expected to be similar for other magic number sized monolayer protected noble metal clusters, but it's unknown if these trends will continue as clusters size increases into the range between cluster and colloidal particle.

\section{Conclusions}

Overall, this data allows us to suggest practical aspects of storage and handling that may preserve the integrity of $\mathrm{Au}_{25}(\mathrm{SR})_{18}$ and by extension other thiolate protected clusters. In general, steric stabilization of the clusters appears to provide 
the greatest tunable variable for manipulating cluster half lives. The nature of the solvent also plays a large and unexpected role in the half-lives of the clusters. Less important but still appreciable contributions are made by oxidation state and relatedly the atmosphere under which clusters are stored. In some conditions, especially when dissolved in toluene with larger ligands the clusters appear to be very stable.

\section{Conflicts of interest}

There are no conflicts to declare.

\section{Acknowledgements}

We thank Eric Knudson for helpful conversations in formulating this work.

\section{Notes and references}

1 M. Walter, J. Akola, O. Lopez-Acevedo, P. D. Jadzinsky, G. Calero, C. J. Ackerson, R. L. Whetten, H. Grönbeck and H. Häkkinen, Proc. Natl. Acad. Sci. U. S. A., 2008, 105, 9157-9162.

2 M. A. Tofanelli and C. J. Ackerson, J. Am. Chem. Soc., 2012, 134, 16937-16940.

3 H. Qian, W. T. Eckenhoff, Y. Zhu, T. Pintauer and R. Jin, J. Am. Chem. Soc., 2010, 132, 8280-8281.

4 P. D. Jadzinsky, G. Calero, C. J. Ackerson, D. A. Bushnell and R. D. Kornberg, Science, 2007, 318, 430-433.

5 M. W. Heaven, A. Dass, P. S. White, K. M. Holt and R. W. Murray, J. Am. Chem. Soc., 2008, 130, 3754-3755.

6 O. Toikkanen, S. Carlsson, A. Dass, G. Rönnholm, N. Kalkkinen and B. M. Quinn, J. Phys. Chem. Lett., 2010, 1, 32-37.

7 A. Desireddy, S. Kumar, J. Guo, M. D. Bolan, W. P. Griffith and T. P. Bigioni, Nanoscale, 2013, 5, 2036-2044.

8 X. Yuan, N. Goswami, I. Mathews, Y. Yu and J. Xie, Nano Res., 2015, 8, 3488-3495.

9 Y. Moroi, in Micelles, Springer US, 1992, pp. 131-148.

10 J. Zhou, J. Ralston, R. Sedev and D. A. Beattie, J. Colloid Interface Sci., 2009, 331, 251-262.

11 Y. Li and M. A. El-Sayed, J. Phys. Chem. B, 2001, 105, 89388943.

12 T. Laaksonen, P. Ahonen, C. Johans and K. Kontturi, ChemPhysChem, 2006, 7, 2143-2149.

13 T. A. Dreier and C. J. Ackerson, Angew. Chem., Int. Ed. Engl., 2015, 54, 9249-9252.
14 B. L. V. Prasad, S. I. Stoeva, C. M. Sorensen and K. J. Klabunde, Chem. Mater., 2003, 15, 935-942.

15 T. G. Schaaff and R. L. Whetten, J. Phys. Chem. B, 1999, 103, 9394-9396.

16 M. Brust, M. Walker, D. Bethell, D. J. Schiffrin and R. Whyman, J. Chem. Soc., Chem. Commun., 1994, 801-802.

17 W. Zhang, in Nanomaterial, ed. D. G. Capco and Y. Chen, Springer Netherlands, 2014, pp. 19-43.

18 M. A. Watzky and R. G. Finke, J. Am. Chem. Soc., 1997, 119, 10382-10400.

19 N. T. K. Thanh, N. Maclean and S. Mahiddine, Chem. Rev., 2014, 114, 7610-7630.

20 E. M. Hotze, T. Phenrat and G. V. Lowry, J. Environ. Qual., 2010, 39, 1909-1924.

21 Y. Shichibu, Y. Negishi, H. Tsunoyama, M. Kanehara, T. Teranishi and T. Tsukuda, Small, 2007, 3, 835-839.

22 J. Akola, M. Walter, R. L. Whetten, H. Häkkinen and H. Grönbeck, J. Am. Chem. Soc., 2008, 130, 3756-3757.

23 S. Antonello, N. V. Perera, M. Ruzzi, J. A. Gascón and F. Maran, J. Am. Chem. Soc., 2013, 135, 15585-15594.

24 M. A. Tofanelli, K. Salorinne, T. W. Ni, S. Malola, B. Newell, B. Phillips, H. Häkkinen and C. J. Ackerson, Chem. Sci., 2016, 7, 1882-1890.

25 J. F. Parker, C. A. Fields-Zinna and R. W. Murray, Acc. Chem. Res., 2010, 43, 1289-1296.

26 Y. Negishi, N. K. Chaki, Y. Shichibu, R. L. Whetten and T. Tsukuda, J. Am. Chem. Soc., 2007, 129, 11322-11323.

27 A. Venzo, S. Antonello, J. A. Gascón, I. Guryanov, R. D. Leapman, N. V. Perera, A. Sousa, M. Zamuner, A. Zanella and F. Maran, Anal. Chem., 2011, 83, 6355-6362.

28 M. Zhu, C. M. Aikens, F. J. Hollander, G. C. Schatz and R. Jin, J. Am. Chem. Soc., 2008, 130, 5883-5885.

29 L.-Y. Chen, C.-W. Wang, Z. Yuan and H.-T. Chang, Anal. Chem., 2015, 87, 216-229.

30 G. Li and R. Jin, Acc. Chem. Res., 2013, 46, 1749-1758.

31 H. Kawasaki, S. Kumar, G. Li, C. Zeng, D. R. Kauffman, J. Yoshimoto, Y. Iwasaki and R. Jin, Chem. Mater., 2014, 26, 2777-2788.

32 A. C. Templeton, S. Chen, S. M. Gross and R. W. Murray, Langmuir, 1999, 15, 66-76.

33 C. J. Ackerson, P. D. Jadzinsky and R. D. Kornberg, J. Am. Chem. Soc., 2005, 127, 6550-6551.

34 D. M. P. Mingos, Inorg. Chem., 1982, 21, 464-466.

35 B. \& J. Laboratories, Solvent guide, Burdick and Jackson Laboratories, 1984. 